\title{
Remote manipulation of magnetic nanoparticles using magnetic field gradient to promote cancer cell death
}

\author{
Mahendran Subramanian ${ }^{1}(10) \cdot$ Arkadiusz Miaskowski $^{2} \cdot$ Stuart lain Jenkins $^{3} \cdot$ Jenson Lim $^{4} \cdot$ Jon Dobson $^{5}$
}

Received: 13 November 2018 / Accepted: 21 February 2019 / Published online: 4 March 2019

(c) The Author(s) 2019

\begin{abstract}
The manipulation of magnetic nanoparticles (MNPs) using an external magnetic field, has been successfully demonstrated in various biomedical applications. Some have utilised this non-invasive external stimulus and there is an potential to build on this platform. The focus of this study is to understand the manipulation of MNPs by a time-varying static magnetic field and how, at different frequencies and displacement, this can alter cellular function. Here we explore, using numerical modeling, the physical mechanism which underlies this process, and we discuss potential improvements for its use in biomedical applications. From our data and other related studies, we infer that such phenomenon largely depends on the magnetic field gradient, magnetic susceptibility and size of the MNPs, magnet array oscillating frequency, viscosity of the medium surrounding MNPs, and distance between the magnetic field source and MNPs. Additionally, we demonstrate cytotoxicity in neuroblastoma (SH-SY5Y) and hepatocellular carcinoma (HepG2) cells in vitro induced by MNPs exposed to a magnetic field gradient and oscillating at various frequencies and displacement amplitudes. Even though this technique reliably produces MNP endocytosis and/or cytotoxicity, a better understanding is required to develop this system for precision manipulation of MNPs, ex vivo.
\end{abstract}

\section{Introduction}

The manipulation of magnetic nanoparticles (MNPs) using external magnetic fields has led to the emergence of novel in vitro technologies. This technique which relies on noninvasive external stimuli, such as gradient magnetic fieldassisted bioseparation [1] and gene transfection [2, 3]; alternating field gradient-mediated cytotoxicity [4]; dynamic

Mahendran Subramanian

m.subramanian@imperial.ac.uk

1 Department of Bioengineering, Department of Computing, Royal School of Mines, Imperial College London, London SW7 2AZ, UK

2 Department of Applied Mathematics and Computer Science, University of Life Sciences, 20-950 Lublin, Poland

3 School of Medicine, Keele University, David Weatherall Building, Staffordshire ST5 5BG, UK

4 Biological and Environmental Sciences, Faculty of Natural Sciences, University of Stirling, Stirling FK9 4LA, UK

5 J. Crayton Pruitt Family Department of Biomedical Engineering, Department of Material Science and Engineering, Institute for Cell and Tissue Science and Engineering, ICTSE, University of Florida, PO Box 116131, Gainesville, FL 32611, USA magnetic field (DMF)-mediated cytotoxicity [5]; alternating magnetic field (AMF)-mediated cytotoxicity [6]; and controlled drug release [7]. The techniques mentioned above involve both permanent magnets and electromagnets with different working principles. Bioseparation consists of the use of a field gradient to capture specific biomolecules which are bound to MNPs [8]. Magnetofection involves the use of a magnetic field to attract magnetic nanoparticles and nucleic acid complexes towards cells to facilitate gene transfection $[9,10]$. The alternating field gradient mediated cytotoxicity technique uses an alternating field gradient $(\mathrm{Gz}-95 \mathrm{G} / \mathrm{cm})$ within a homogenous field (a $9.4 \mathrm{~T}$ preclinical MRI system) to align the nanoparticles parallel to the homogenous field and destroy cancer cells through an induced motion of magnetic nanoparticle aggregates within cells [4]. Dynamic magnetic fields $(10-20 \mathrm{~Hz}, 30 \mathrm{mT})$ encourage torques, i.e., repeated incomplete rotational movements, of MNPs which enables remote stimulation of cell death by permeabilising the lysosome membrane and triggering apoptosis (programmed cell death) [5]. The application of alternating magnetic fields (50-1000 kHz) to MNPs which are bound to receptors on cell membranes or internalized within cells, has been used to activate chemical signaling in the cell, leading to apoptosis or the depositing of energy, triggering necrosis (cell death or tissue damage in an organ) [11]. 
The possible physical explanations for the static magnetic field gradient-mediated attracting/aligning of MNPs towards a magnet field have been discussed extensively [12-15]. Moreover, a possible mechanism for alternating field gradient mediated cytotoxicity has been examined [4]. However, an explanation for dynamic field-induced rotational motion has proven elusive; it is stated in [4] that a dynamic field generates a torque, equal to and that this enables the rotation of individual MNPs around their axis, but the dynamics of the magnetic fields used requires further exploration. Moreover, deposition of energy is possible when MNPs are exposed to alternating magnetic fields in the radio frequency range, since they dissipate heat due to susceptibility, hysteresis and friction losses [12].

This study focuses on the underlying conditions behind the manipulation of MNPs using unidirectional time-varying magnetic fields/field gradients and how this can induce magneto-mechanical cell death in cancer cells. The numerical model discussed here will direct researchers to this novel technique. Here, we used a time-varying $(1-4 \mathrm{~Hz})$ alternate pole magnet array plate populated with $\mathrm{Nd}-\mathrm{Fe}-\mathrm{B}$ permanent magnets $(\sim 450 \mathrm{mT})$ to create field gradients which result in enhanced attraction of magnetic nanoparticle towards cells which led to cytotoxicity in neuroblastoma (SH-SY5Y) and hepatocellular carcinoma (Hep G2) cancer cells.

\section{Materials and methods}

\subsection{Magnetic field measurements}

Magnetic field measurements of three different $3 \times 3$ $\mathrm{Nd}-\mathrm{Fe}-\mathrm{B}$ magnet arrays were performed using a Hall probe (F.W.Bell-5080 Teslameter; Orlando, Florida, USA). The numerical modeling discussed in this paper was carried out using the Sim4Life (ZMT Zurich MedTech, Zurich, Switzerland) platform. The dimensions of the magnets and the corresponding distances were measured in relation to the magnet array which was explicitly fabricated for performing the experiments here.

\subsection{Mathematical model}

These measurements were used to calculate the magnetic field (flux density, $T$ ) of each $3 \times 3$ array (Fig. 1), the magnetic force $(N)$ experienced by the MNPs, and the gradient field (gradient flux density; $\mathrm{T} / \mathrm{m}$ ) for specific distances from the magnet faces along the $z$-axis $(0 \mathrm{~mm}, 1 \mathrm{~mm}, 1.5 \mathrm{~mm})$ and from the centre of each magnet along the $y$-axis $(-3$ to $+3 \mathrm{~mm} ; 2 \mathrm{D}$ plot).

The magnetic field was calculated using the magnetic vector potential $(\boldsymbol{A})$ under the assumption that $\nabla \cdot(\boldsymbol{B})=0$ and $B=\nabla \times(A)$, i.e.: $\nabla \times \frac{1}{\mu} \nabla \times \boldsymbol{A}=J_{0}$

where $J_{0}$ is the surface current density $(\mathrm{A} / \mathrm{m})$.

In addition, to explain the aforementioned phenomenon (magneto-mechanical cell death), the following mathematical model can be used.

The magnetic moment, $\mathbf{m}$, of a nanoparticle (based on the typical design of having magnetic material at the core) is a product of its magnetization, $\mathbf{M}$, and volume, $V_{\mathrm{m}}=\frac{4}{3} \pi R_{\mathrm{m}}^{3}$, where $R_{\mathrm{m}}$ is the radius of its magnetic core:

$\boldsymbol{m}=\boldsymbol{M} V_{\mathrm{m}}$.

The volumetric magnetization of the MNPs is induced by application of the external magnetic field, $\boldsymbol{H}$,

$M=\Delta \chi H$,

where $\Delta \chi$ is the effective susceptibility of the MNPs with respect to the medium (water) and:

$\Delta \chi=\chi_{\mathrm{MNP}}-\chi_{\text {water }}$.

Assuming that the relative permeability of the water, $\mu_{\mathrm{r}}=1$, together with $\boldsymbol{B}=\mu_{0} \boldsymbol{H}$, the volumetric magnetization can be introduced as:

$M=\frac{\Delta \chi B}{\mu_{0}}$,

and

$m=\frac{\Delta \chi V_{m} B}{\mu_{0}}$.

Furthermore, if MNPs are point dipoles with the same moment, $\mathbf{m}$, the force experienced by them is

$\boldsymbol{F}_{m}=(\boldsymbol{m} \cdot \nabla) \boldsymbol{B}$.

Combining (6) and (7), we get
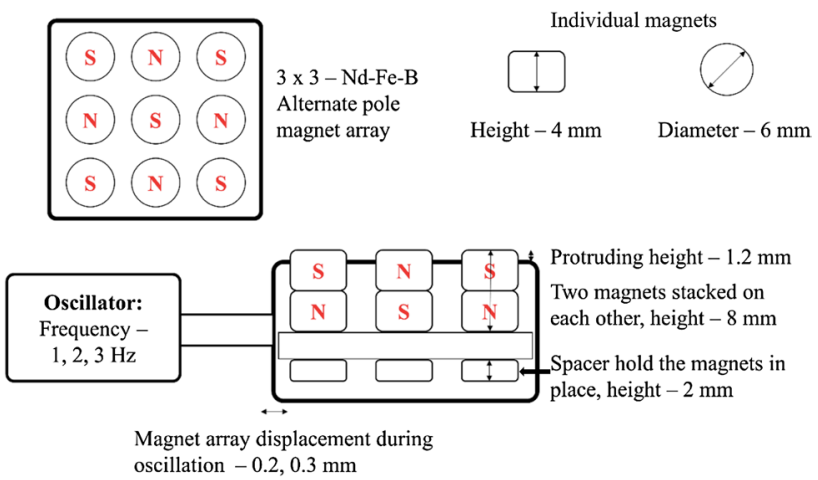

Fig. 1 Illustration of the alternate pole magnet array, made up of $\mathrm{Nd}-$ $\mathrm{Fe}-\mathrm{B}$ permanent rare earth magnets, which was used for the induction of magneto-mechanical cytotoxicity in cancer cells. Dimensions of the magnet used (height, diameter) and the populated magnet array (cross section) connected to the oscillator are provided 
$F_{m}=(m \cdot \nabla) B=\frac{V_{m} \Delta \chi}{\mu_{0}}(B \cdot \nabla) B$.

Knowing that $\nabla \times \boldsymbol{B}=0$, the force on the dipole can be modified as:

$F_{m}=\frac{V_{m} \Delta \chi}{2 \mu_{0}} \nabla(B \cdot B)=\frac{V_{m} \Delta \chi}{2 \mu_{0}} \nabla B^{2}=V_{m} \Delta \nabla\left(\frac{1}{2} B \cdot H\right)$.

To be effective, $\boldsymbol{F}_{\mathrm{m}}$ must overcome the hydrodynamic drag force, $\boldsymbol{F}_{\mathrm{d}}$, acting on the MNPs, i.e.,

$F_{d}=6 \pi \eta R_{h} \Delta v_{m}$,

where $\eta$ is the viscosity of the medium (water) surrounding the MNPs, $R_{\mathrm{h}}$ is the hydrodynamic radius, and $v_{\mathrm{m}}$ is the drift velocity $\left(\Delta v_{m}=v_{\text {MNP }}-v_{\text {water }}\right)$. The MNPs will be immobilized when $\boldsymbol{F}_{\mathrm{d}}=\boldsymbol{F}_{\mathrm{m}}$, so:

$6 \pi \eta R_{h} \Delta v_{m}=\frac{4 R_{m}^{3} \Delta \chi}{6 \mu_{0}} \nabla B^{2}$,

or

$\Delta v_{m}=\frac{R_{m}^{3} \Delta \chi}{9 \mu_{0} \eta R_{h}} \nabla B^{2}=\xi \nabla B^{2}$,

where $\xi$ is the magnetophoretic mobility.

As the first approximation, it can be assumed that $R_{\mathrm{m}}=R_{\mathrm{h}}$ and when

$\xi=\frac{R_{m}^{2} \Delta \chi}{9 \mu_{0} \eta}$

As the second approximation, it can be assumed that $R_{\mathrm{m}} \neq R_{\mathrm{h}}$ and when

$\xi=\frac{R_{m}^{3} \Delta \chi}{9 \mu_{0} \eta R_{h}}$,

where $V_{\mathrm{h}}=\left(1+\frac{\delta}{R_{\mathrm{m}}}\right)^{3} V_{\mathrm{m}}$ and $\delta$ is the thickness of the absorbed surface layer.

\subsection{Cell culture}

Human neuroblastoma cells (SH-SY5Y; CRL-2266; American Type Culture Collection, Manassas, USA) were maintained as described previously [16]. SH-SY5Y cells were seeded at a density of 1 or $2 \times 10^{4}$ cells in $100 \mu \mathrm{l}$ medium onto uncoated 96-well plates in Ham's F12: MEM (1:1) supplemented with $10 \%$ fetal bovine serum (FBS), $2 \mathrm{mM}$ L-glutamine, $100 \mathrm{U}$ penicillin, and $0.1 \mathrm{mg} / \mathrm{ml}$ streptomycin. Human hepatocellular carcinoma cells (Hep G2; HB-8065; American Type Culture Collection, Manassas, USA) were cultured in DMEM with 10\% FBS, 2 mM L-glutamine, 100 $\mathrm{U}$ penicillin, and $0.1 \mathrm{mg} / \mathrm{ml}$ streptomycin, prior to seeding at a density of 1 or $2 \times 10^{4}$ cells in $100 \mu \mathrm{l}$ medium onto rat tail collagen I-coated 96-well plates. Cultured cells were incubated at $37{ }^{\circ} \mathrm{C}, 5 \% \mathrm{CO}_{2}$, for $24 \mathrm{~h}$ prior to cytotoxicity experiments.

\subsection{Magnetic field gradient-mediated cytotoxicity experiments}

Magnetite nanoparticles were obtained from Ozbiosciences, Marseilles, France (Polymag MNPs, used with SH-SY5Y cells) and nanoTherics Ltd, Staffordshire, UK (nTmag MNPs, used with Hep G2 cells). Both Polymag and nTmag contains a magnetite core. They range between 100 and $250 \mathrm{~nm}$ in hydrodynamic diameter and are surface functionalized with proprietary polyethyleneimine derivative. Both have positive zeta potential (surface charge). The rationale for using both particles is that the study was aimed at evaluation of MNP + magnet array-mediated cancer cytotoxicity. Our goal was to demonstrate that the technology works efficiently with different commercially available magnetic nanoparticles that have been widely demonstrated to show high biocompatibility [10,17]. MNPs were diluted in $100 \mu \mathrm{l}$ medium and added to cell cultures $(0.2 \mu \mathrm{l}$ per well) immediately prior to magnetic field application. Cell culture plates were placed over the magnet array, with cells $1 \mathrm{~mm}$ above magnet surface, along the $z$-axis. Magnet array oscillated along the $x$-axis using a stepper motor for 30 min with the indicated frequencies and displacements. The experiment was conducted within a $37{ }^{\circ} \mathrm{C}, 5 \% \mathrm{CO}_{2}$, tissue culture incubator during the 30 -min exposure.

\subsection{Cell viability assay}

After exposure to the oscillating magnet array, cell culture plates were returned to the incubator for $48 \mathrm{~h}$ before viability testing. The CytoTox-ONE ${ }^{\mathrm{TM}}$ membrane integrity assay (Promega, Southampton, UK) provided a measurement of the amount of lactate dehydrogenase (LDH) released through damage to cell membranes. The assay was performed according to the manufacturer's instructions, and luminescence was recorded using a plate reader (BioTek, Bedfordshire, UK).

$\%$ viability $=100-\left[100\left[\frac{\text { experimental }- \text { backgrond luminescence }}{\text { maximum LDH release }- \text { background luminescence }}\right]\right]$ 


\subsection{Magnetic nanoparticle uptake}

$0.2 \mu$ Polymag or $0.1 \mu \mathrm{lnTmag}$ nanoparticles were incubated with $0.2 \mu \mathrm{g}$ or $0.3 \mu \mathrm{g}$ pEGFPN1 plasmid (encoding the green fluorescent protein, GFP) for $15 \mathrm{~min}$, then added to cultures which were immediately placed on the magnet array (30 min oscillation at $2 \mathrm{~Hz}$ frequency, $0.2 \mathrm{~mm}$ horizontal displacement). GFP expression indicates cellular uptake of nanoparticles and expression of the plasmid. Fluorescence was observed after $48 \mathrm{~h}$ incubation using a Leica DM IL LED fluorescent microscope (Newcastle Upon Tyne, UK) or Nikon fluorescent microscope (Amsterdam, Netherlands).

\subsection{Statistics}

If not stated otherwise, the data were presented as mean $\pm \mathrm{SD}$ of values from at least three experiments. Statistical significance was determined by a one-way ANOVA followed by a Bonferroni post hoc test (multiple comparison test) with $p>0.05$ considered as not significant.

\section{Results}

The magnetic field surrounding an array of magnets was measured to inform a theoretical prediction of magnetic nanoparticle behavior within such a field. Cancer cell lines were exposed to magnetic nanoparticles in the presence of an oscillating magnetic field gradient to assess their potential for inducing cytotoxicity.

\subsection{Magnetic field measurements}

Magnetic field (flux density) measurements above the magnet array were determined at the magnet face, and base of a culture plate immediately above the array (see Fig. 2).

\subsection{Numerical modeling}

Based on the magnetic field measurements shown in Fig. 2, magnetic field distribution and gradient flux density calculations were performed for the magnet array set up used for conducting experiments. These estimates provide insight into the principles behind the manipulation of MNPs.

The magnetic fields and the field gradients produced by the magnet array were calculated ( $x-, y-, z$-axes). The magnetic force experienced by the MNPs and the velocity of the MNPs relative to the carrier fluid were calculated using the numerical model described in the methods section. Contour plots of the parameters mentioned above for $1 \mathrm{~mm}$ above the magnet array ( $z$-axis) provide a better understanding, aiding in the positioning of the cells and MNPs (see Figs. 3, 4).
Field gradient decreases with distance ( $z$-axis) from the magnet face (Fig. 4). Here, it is important to note that the internal diameter of a cell culture well is $6.5 \mathrm{~mm}$ (typical 96 well plate). Figure $4 \mathrm{~b}$ and $2 \mathrm{~d}$ plot in 4 (c) demonstrate that the magnetic flux gradient is relatively low in the center and higher near the magnet's edge.

Field gradient graphs showing $x$-axis against $y$-axis (horizontal magnet displacement during array oscillation: $0 \mathrm{~mm}$ and $0.1 \mathrm{~mm}$ ) should provide us with insights into the changes occurring in the magnetic field and field gradient when the magnet array is moved back and forth along the $x$-axis (see Fig. 5). The magnetic field flux (Fig. 5a) and field gradient data (Fig. 5b) demonstrate that the magnetic field shifts in the $y$-axis over $0-0.1 \mathrm{~mm}$, a relatively short distance.

The magnetic force, $\left(\boldsymbol{F}_{m}\right)$, and the velocity of the MNPs will be directly proportional to the field gradient $\left(\mathbf{B}^{2}\right)$ : i.e., they will appear as a crater in 3D at $1 \mathrm{~mm}$ ( $z$-axis). And, the displacement of the MNPs will be inversely proportional to the time-varying frequency.

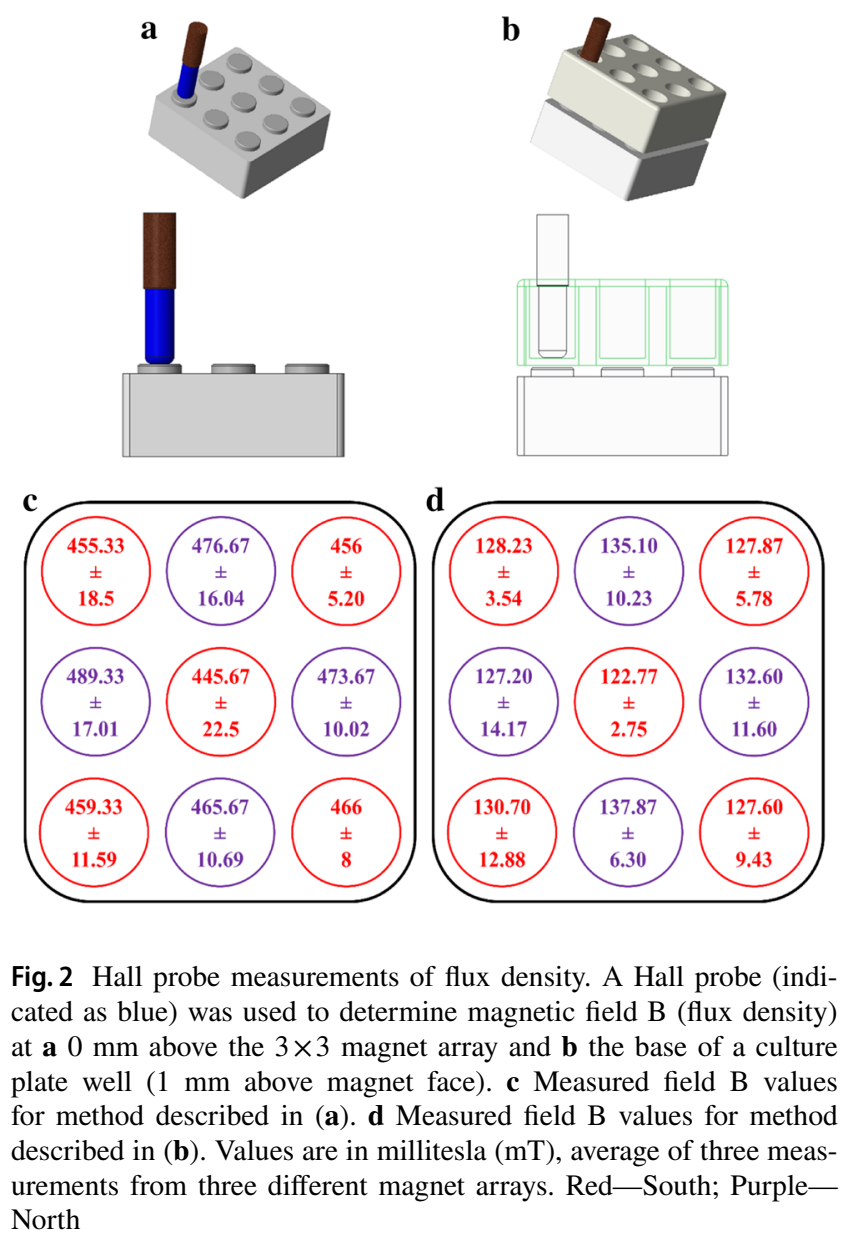

Fig. 2 Hall probe measurements of flux density. A Hall probe (indicated as blue) was used to determine magnetic field B (flux density) a $0 \mathrm{~mm}$ above the $3 \times 3$ magnet array and $\mathbf{b}$ the base of a culture for method described in (a), d Measured field B values for method described in (b). Values are in millitesla (mT), average of three measNorth 
Fig. 3 2D model of flux density above magnetic array. A magneto-static (vector potential) algorithm was used to calculate: (a) magnetic field (flux density) distribution over the $3 \times 3$ magnet array at $1 \mathrm{~mm}$ above the magnets; and (b) magnetic field (flux density) distribution at 0.5 , $1,1.5 \mathrm{~mm}$ above the magnets (z-axis). 3 and $-3 \mathrm{~mm}$ distance represent magnet edges. Legend shows $(x, y, z$ coordinates in $\mathrm{mm})$
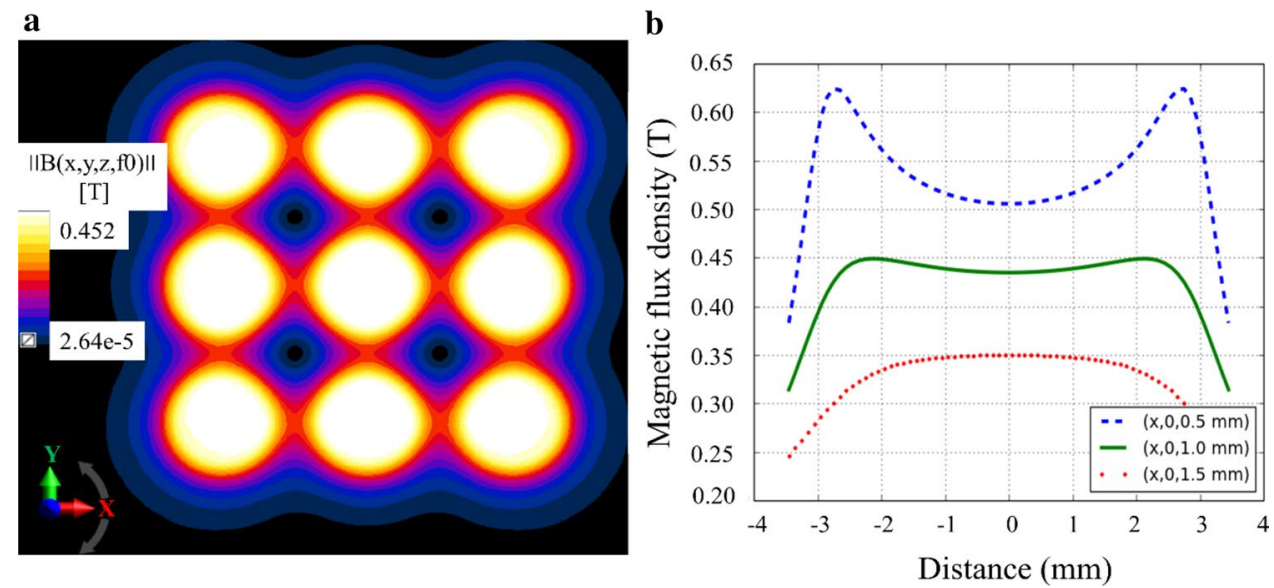

a
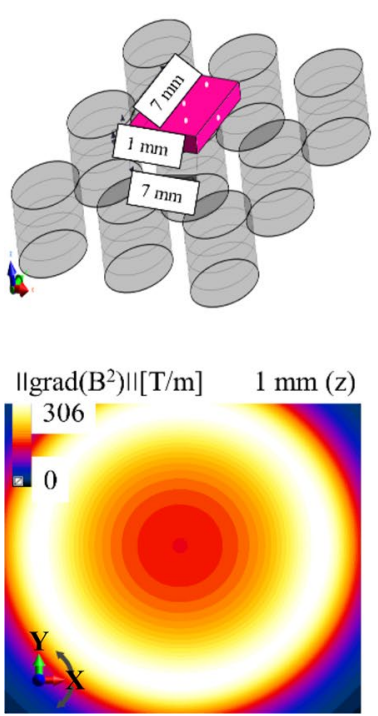

b

$\left\|\operatorname{grad}\left(\mathrm{B}^{2}\right)\right\|[\mathrm{T} / \mathrm{m}] \quad 0.5 \mathrm{~mm}(\mathrm{z})$

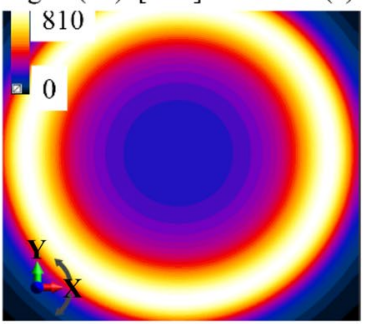

$\left\|\operatorname{grad}\left(\mathrm{B}^{2}\right)\right\|[\mathrm{T} / \mathrm{m}] \quad 1.5 \mathrm{~mm}(\mathrm{z})$

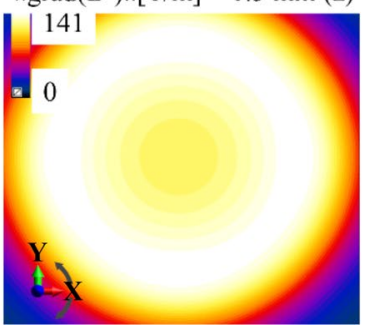

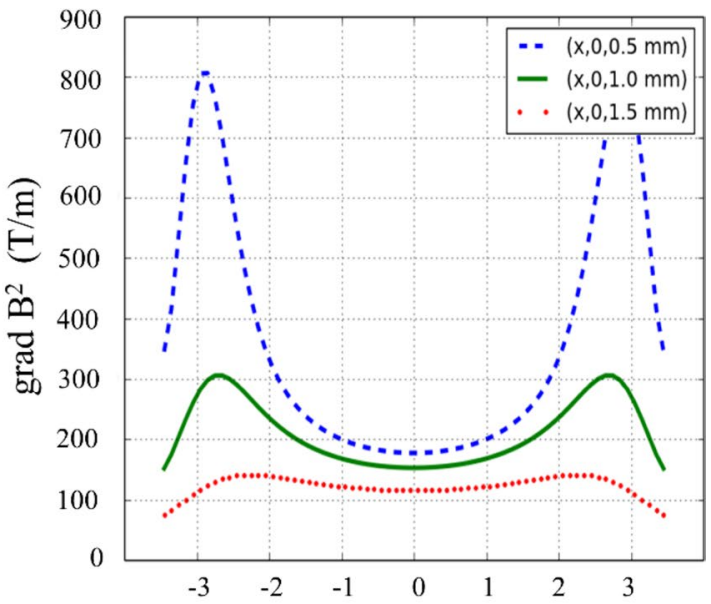

Distance $(\mathrm{mm})$
Fig. 4 Modeling of gradient flux density above a static magnet. a 3D model of the $3 \times 3$ magnet array used for gradient flux density calculations. $\mathbf{b}$ The field gradient distribution (gradient flux density) at 0.5 , 1 , and $1.5 \mathrm{~mm}$ along the $z$-axis (vertically above centre of magnet,

\subsection{Cytotoxicity of neuroblastoma cancer cells}

Cytotoxicity was successfully induced in both cancer cell lines following exposure to magnetic nanoparticles and an oscillating magnetic array. Level of cytotoxicity was dependent on frequency and displacement of the magnet arrays determined by membrane integrity assay (Fig. 6). Viability decreased with increasing oscillating frequency and increasing amplitude of array oscillation. Polymag MNPs, with $3 \mathrm{~Hz} / 0.3 \mathrm{~mm}$ oscillation reduced viability to $70.5 \pm 6.0 \%$. $x=y=0$ ). c $2 \mathrm{D}$ plot of the field gradient distribution (gradient flux density) across the midline of a magnet at $0.5,1$ and $1.5 \mathrm{~mm}$ above the magnet face

\subsection{Nanoparticles were endocytosed by neuroblastoma cells}

To establish that the nanoparticles were endocytosed by neuroblastoma cells, Polymag nanoparticles were coated with plasmids encoding GFP, before incubation with cells, in the presence of a magnetic array. Control cells were included (plasmid only, no MNPs) and exposed to the magnetic field. Green fluorescence was observed by cells which had endocytosed MNPs and subsequently expressed GFP from the adsorbed plasmid (Fig. 7a). As expected, GFP expression was not observed in control cells. There was negligible uptake with the control containing plasmid 


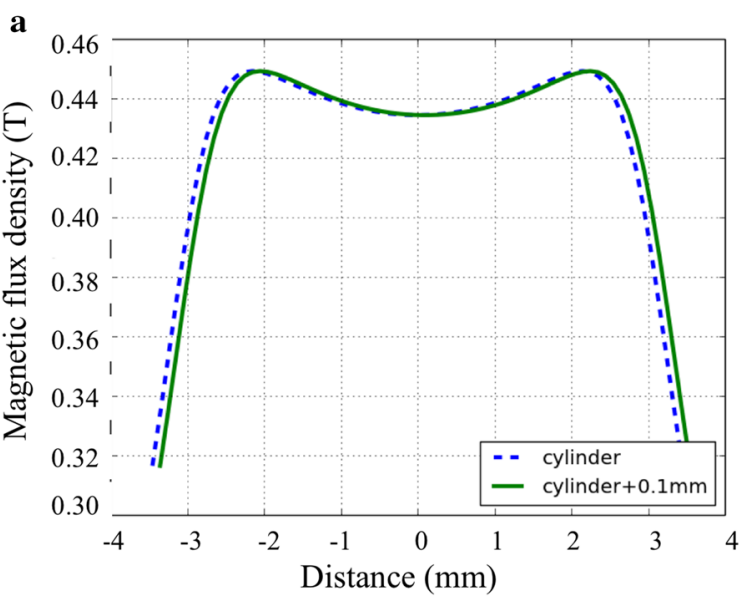

Fig. 5 Gradient flux density at culture well surface (adherent cell layer) during horizontal oscillation of the magnetic array. a 2D plot of the magnetic field (flux density) for $0 \mathrm{~mm}$ ( $x$-axis) and $0.1 \mathrm{~mm}$ shifted

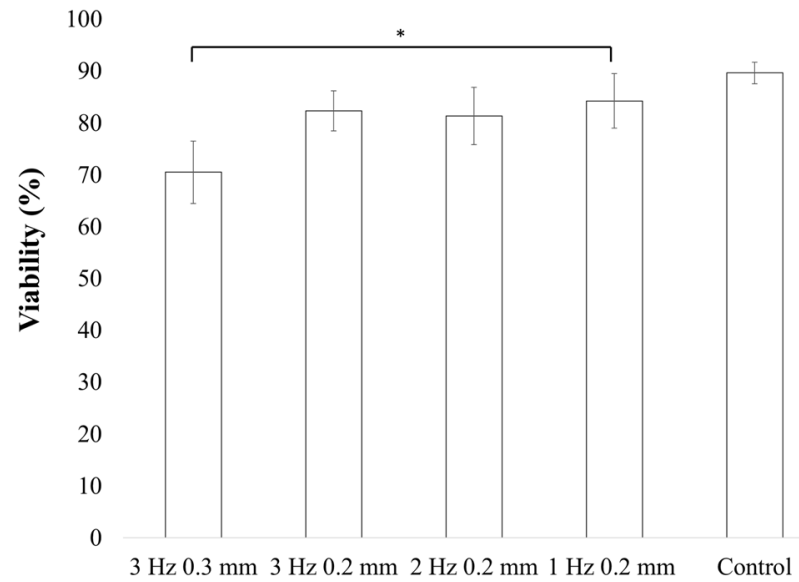

Fig. 6 Magneto-mechanically induced cytotoxicity in SHSY-5Y neuroblastoma cells, exposed to magnetic nanoparticles and a time-varying magnetic field gradient. $n=3$. Control-MNPs only and no magnetic field. Asterisk denotes $P \leq 0.05$

only. This demonstrates that the cytotoxicity observed (Fig. 6) can be attributed to internalization of MNPs.

\subsection{Cytotoxicity of hepatocellular carcinoma cells}

At the highest oscillation frequency tested $(4 \mathrm{~Hz})$, viability of Hep G2 cells in the presence of MNPs had the lowest viability $(53.1 \pm 11.4 \%$; Fig. 8$)$.

\subsection{Nanoparticles were endocytosed by hepatocellular carcinoma cells}

GFP expression by Hep G2 carcinoma cells indicated endocytosis of MNPs and subsequent plasmid expression b

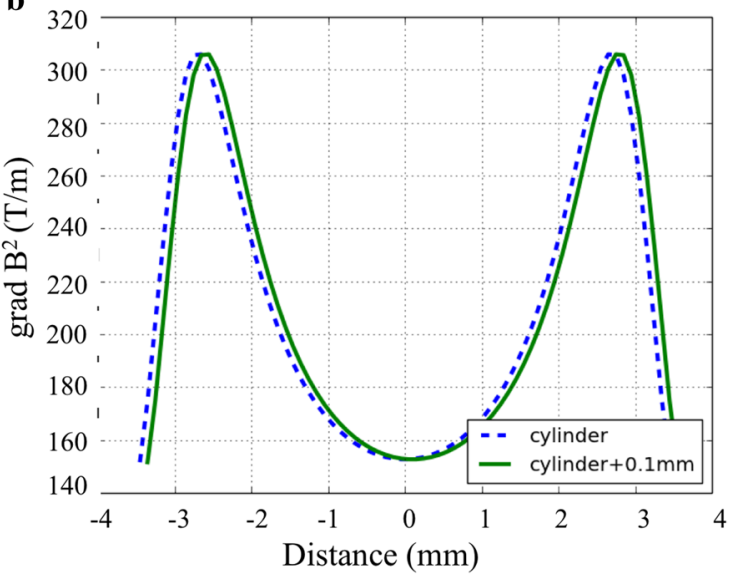

along the $y$-axis. $\mathbf{b}$ The field gradient (gradient flux density) for $0 \mathrm{~mm}$ ( $x$-axis) and $0.1 \mathrm{~mm}$ shifted along the $y$-axis

(Fig. 9a). As before, GFP expression was not observed in control cultures and there was negligible uptake with control cells containing plasmid only (no MNPs). This demonstrates that the cytotoxicity observed (Fig. 8) can be attributed to internalization of MNPs.

\subsection{Magnet array optimization}

Based on the observed results, we propose that magnet array design could be improved to enhance performance when delivering MNPs to a cell monolayer within a round culture well. To address this goal, we evaluated different magnet shapes and arrangements using our model, with the aim of identifying improved field gradient experienced by MNPs/ cells. Two different types of magnet array were compared with the magnet array used in this study via numerical modeling. A magnet array made up of pyramidal magnets, and a custom-shaped Halbach magnet array based on [18, 19] were taken into consideration (Fig. 10). The magneto-static algorithm described earlier was used to calculate magnetic field distributions (Fig. 11) for the magnet arrays shown in Fig. 10. The field strength values at $1 \mathrm{~mm}$ above the magnet apex ( $z$-axis) were used to calculate the field gradient.

From Fig. 12, we can infer that the gradient field values at $1 \mathrm{~mm}$ and $1.5 \mathrm{~mm}$ ( $z$-axis) were higher for the magnet array made up of pyramid-shaped magnets and optimized Halbach magnet array than the magnet array used in this study. Optimized Halbach array produced better gradient field out of all three designs. Moreover, $\boldsymbol{F}_{\mathrm{m}}$ is directly proportional to the gradient magnetic field (see Eq. 9). These numerical calculations shown in Fig. 12 demonstrate that prospects are available for further magnet array optimization to enhance the manipulation of magnetic nanoparticles. 


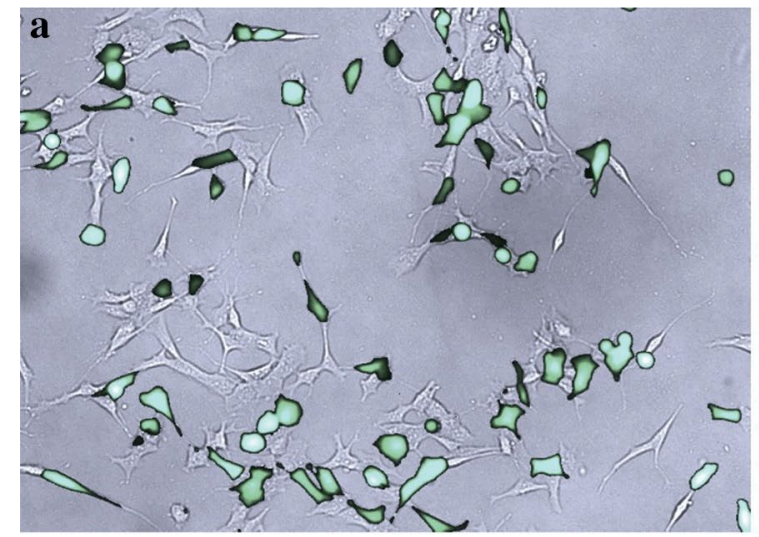

Fig. 7 Endocytosis of Polymag MNPs by SHSY-5Y cells was confirmed by GFP expression. a Fluorescence micrograph of GFP + SHSY-5Y cells superimposed on counterpart phase contrast micrograph, demonstrating expression of a GFP-encoding plasmid carried by Polymag MNPs (with oscillating magnetic field, fre-

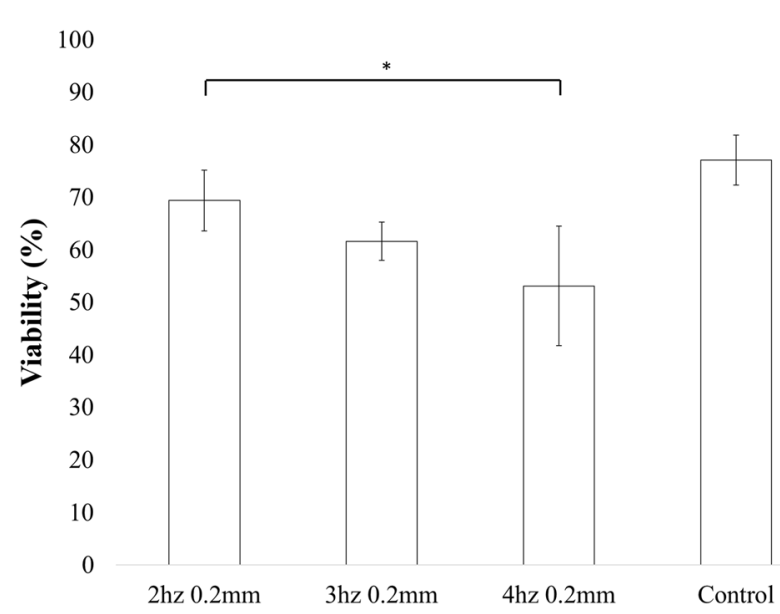

Fig. 8 Magneto-mechanically-induced cytotoxicity in Hep G2 carcinoma cells, exposed to magnetic nanoparticles and a time-varying magnetic field gradient. $n=3$. Control-MNPs only and no magnetic field. Asterisk denotes $P \leq 0.05$

\section{Discussion}

To understand the mechanism behind the observed cytotoxicity mediated by MNPs, it is essential to study the magnetic field and the field gradients produced by the magnet array. Previous studies on a static magnetic array suggest that the magnet does accelerate the sedimentation of MNPs onto adherent cells by overcoming diffusion-limited accumulation [13]. This sedimentation rate can be influenced by varying the size of the MNPs and/or the distance between the magnet array and the cell culture plate [13]. Furthermore, a stronger axial magnetic force, achieved using an alternating magnetic pole array rather than a unidirectional

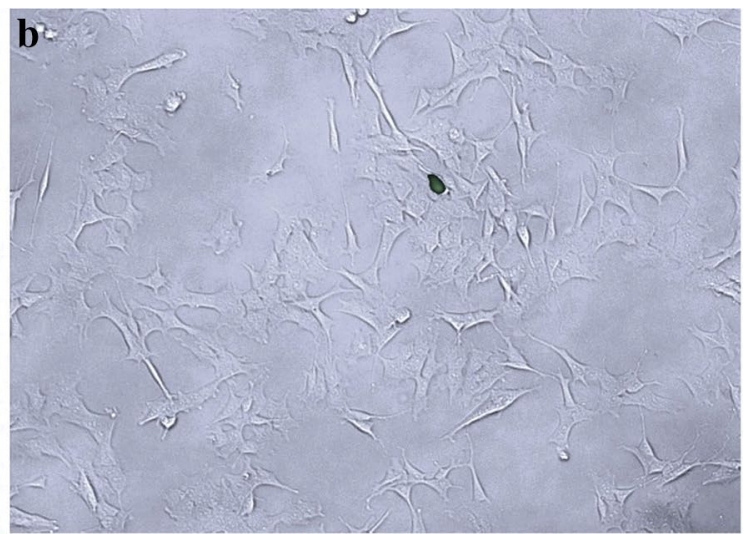

quency $=2 \mathrm{~Hz}$, amplitude $=0.2 \mathrm{~mm}$ ). b Fluorescence micrograph of GFP- SHSY-5Y cells superimposed on counterpart phase contrast micrograph, demonstrating absence of expression of GFP when incubated with plasmid without Polymag MNPs as a vector (with oscillating magnetic field, frequency $=2 \mathrm{~Hz}$, amplitude $=0.2 \mathrm{~mm}$ )

magnet array, improves the accumulation rate [14]. There are some interesting questions posed by the results of the present study regarding the mechanism behind this unique biophysical technique which facilitates the damage of cells via a laterally oscillating high gradient magnet array. Based on our model, the field gradient should be considered the most dynamic variable when calculating the $\boldsymbol{F}_{\mathrm{m}}(N)$ from (9). Thus, high field gradient will increase the force acting upon the magnetic nanoparticles. Assuming that the magnetic array will move without any drag or delay in the field over a displacement distance of $0.2 \mathrm{~mm}$.

A scrutiny of the magnetic flux density $(B)$, its squared gradient $\left(B^{2}\right)$, the magnetic force $\left(\boldsymbol{F}_{\mathrm{m}}\right)$ and the velocity of the MNPs (Figs. 2, 3, 4, 5 and numerical calculations) suggests that these values vary within a single well (internal diameter $-6.5 \mathrm{~mm}$ ) of the (static) cell culture vessel when an alternating-pole magnet array is moved back and forth underneath. It is already evident that the $\boldsymbol{F}_{\mathrm{m}}$ overcomes some of the viscous drag force of culture medium, since the application of this alternating-pole magnet array resulted in the accumulation of $90 \%$ of MNPs at the bottom of the plate after $20 \mathrm{~min}$ of exposure to a static array [14]. When most of the particles are in contact with the monolayer of cultured cells, the nanoparticles will experience a field gradient and a homogenous field (Figs. 3, 4, 5). MNPs in the gradient field will experience a translational motion and in the homogeneous field will experience a rotational motion-in addition to the random Brownian movement (walk) [12]. The magnetophoretic parameter $(\xi)$, i.e., the manipulability of $100 \mathrm{~nm}$ (HD)-sized MNPs used in this study, can be calculated using (14), and the displacement values resulting from the velocity do predict that the nanoparticles undergo a translational movement. 


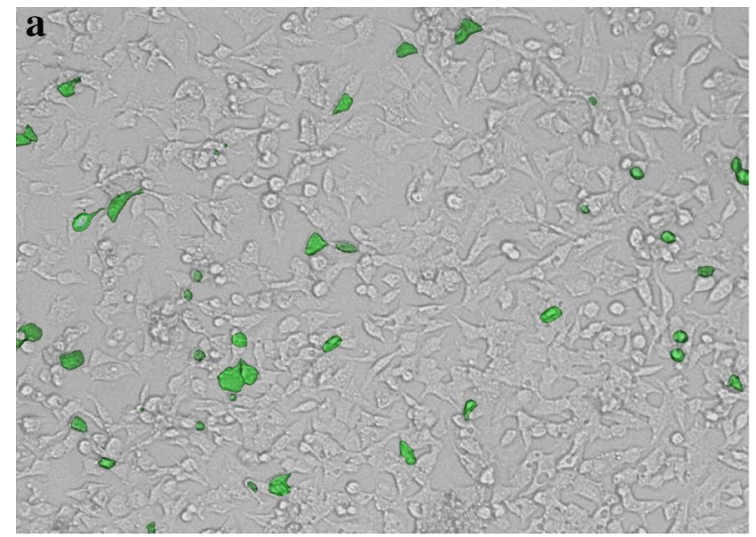

Fig. 9 Endocytosis of nTmag MNPs by Hep G2 carcinoma cells was confirmed by GFP expression. a Fluorescence micrograph of GFP + Hep G2 cells superimposed on counterpart phase contrast micrograph, demonstrating expression of a GFP-encoding plasmid carried by nTmag MNPs (with oscillating magnetic field, fre-

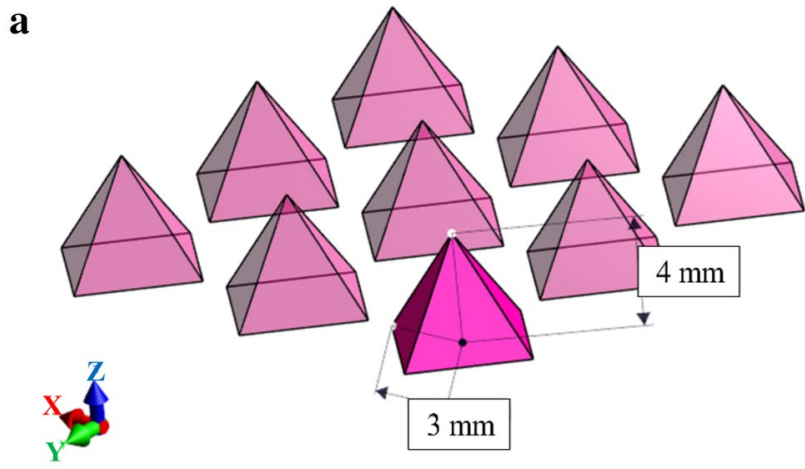

b

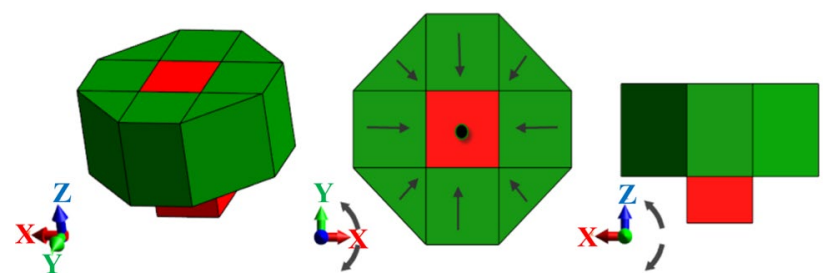

Fig. 10 Proposed magnet array with pyramid-shaped magnets (a); optimized Halbach magnet array (b)

Hence, we can advocate from our calculations that the MNPs will experience oscillating horizontal translational motion when the magnet array is moved laterally $(-0.1$ to $0.1 \mathrm{~mm}$ in the $x$-axis for $0.2 \mathrm{~mm}$ displacement or -0.15 to $0.15 \mathrm{~mm}$ for $0.3 \mathrm{~mm}$ displacement) underneath the cell culture plate. And this displacement of MNPs is inversely proportional to the time-varying frequency of the magnet array movement. In other words, the translational movement of the MNPs, induced by the time-varying magnet array will

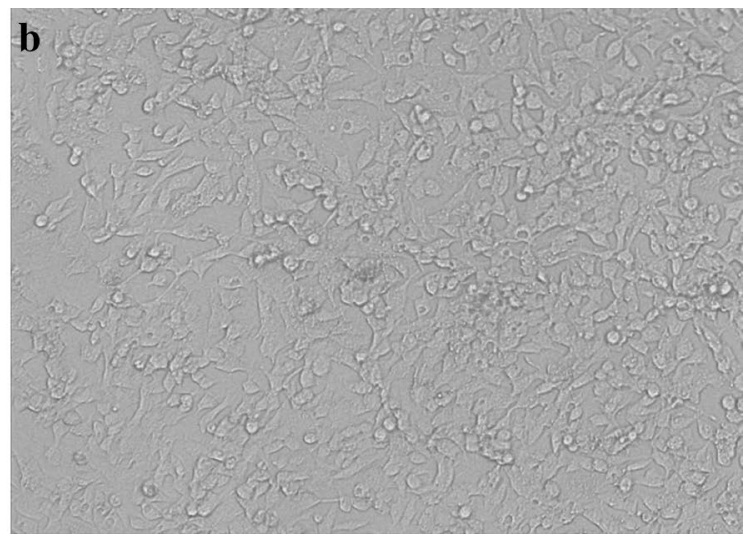

quency $=2 \mathrm{~Hz}$, amplitude $=0.2 \mathrm{~mm}$ ). b Fluorescence micrograph of GFP-Hep G2 cells superimposed on counterpart phase contrast micrograph, demonstrating absence of expression of GFP when incubated with plasmid without nTmag MNPs as a vector (with oscillating magnetic field, frequency $=2 \mathrm{~Hz}$, amplitude $=0.2 \mathrm{~mm}$ )

reduce, if we increase the time-varying frequency. But, the number of movement cycles will increase.

An important point to note is that MNPs tend to form aggregates, and this may have an impact on the translational motion of the MNPs [4]. The low displacement values may be due to viscous damping and displacement might be higher when the nanoparticles form aggregates. However, this back and forth motion, through the influence of a time-varying field gradient, should have induced mechanical stress on the surface/inside of the cells, which in turn should increase cell death; this effect is facilitated by the magnet's displacement. Even though the displacement values are low, the mechanical manipulation would cause stress for 30-min exposure at $2 \mathrm{~Hz}$, as the total number of oscillations would be 3600 , i.e., 120 cycles per minute. And, we should not exclude nanoparticle clusters. The sizes of animal cells are usually between 0.01 and $0.1 \mathrm{~mm}$, and cell cytoplasm has a higher viscosity than the surrounding cell culture medium. So, gradient magnetic field-assisted movement of MNPs should reduce once the MNPs are endocytosed by cells; however, there are studies validating the motility of small molecules within cytoplasm [20].

This spatio-temporal behavior parameter, i.e., the oscillating motion of the MNPs, must be responsible for the increase in the cytotoxicity of the neuroblastoma cancer cells. In vitro numerical investigation suggests that magnet geometry influences the collection time of the magnetic particles moving through a high viscosity fluid. Furthermore, we use cylindrical shape magnets with an aspect ratio of 1:1.66; this is comparable to the magnet geometry used for in vivo studies; this is cylindrical—not conical—and has an aspect ratio of 1:1 [15]. Gradient magnetic field being the most dominant parameter to manipulate MNPs in vitro and 


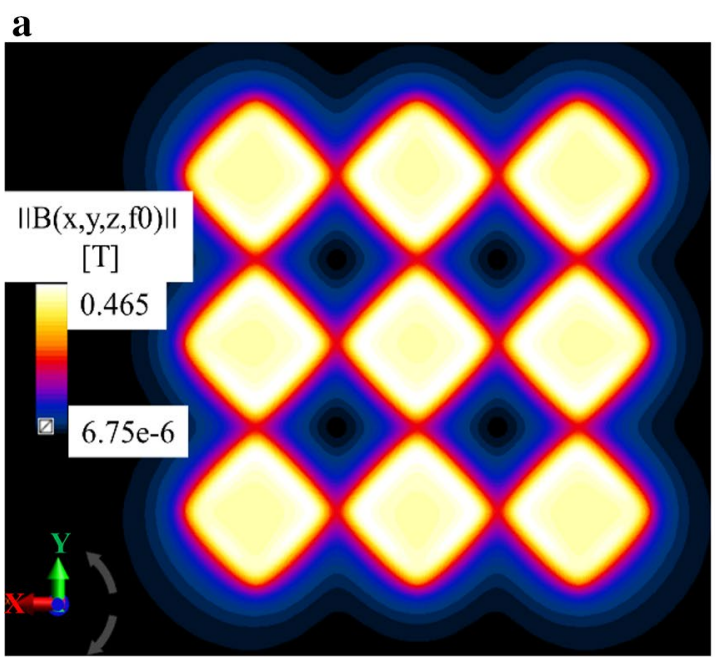

Fig. 11 Magneto-static (vector potential) algorithm-based numerical calculations: (a) magnetic field (flux density) distribution over the $3 \times 3$ magnet array (pyramid shaped magnets) at $1 \mathrm{~mm}$ above the b

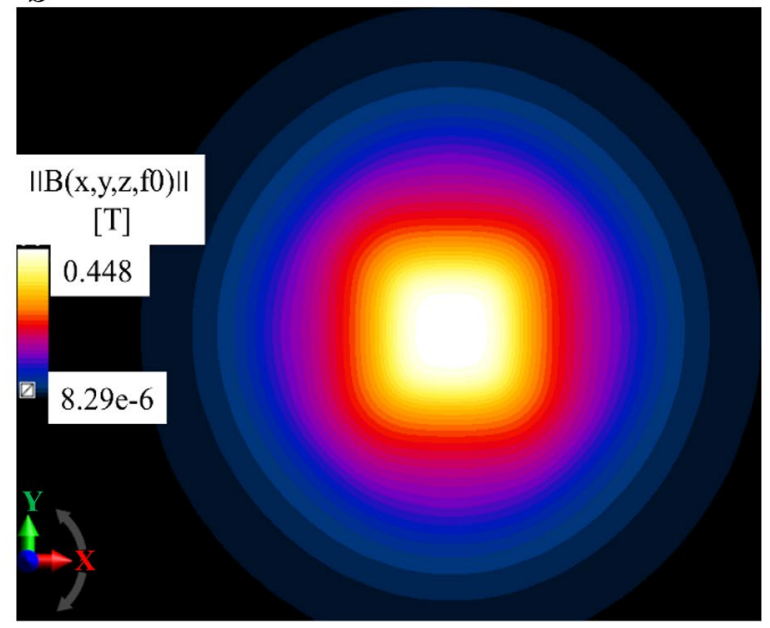

magnets (b) magnetic field (flux density) distribution over the optimized Halbach magnet array at $1 \mathrm{~mm}$ above the magnets
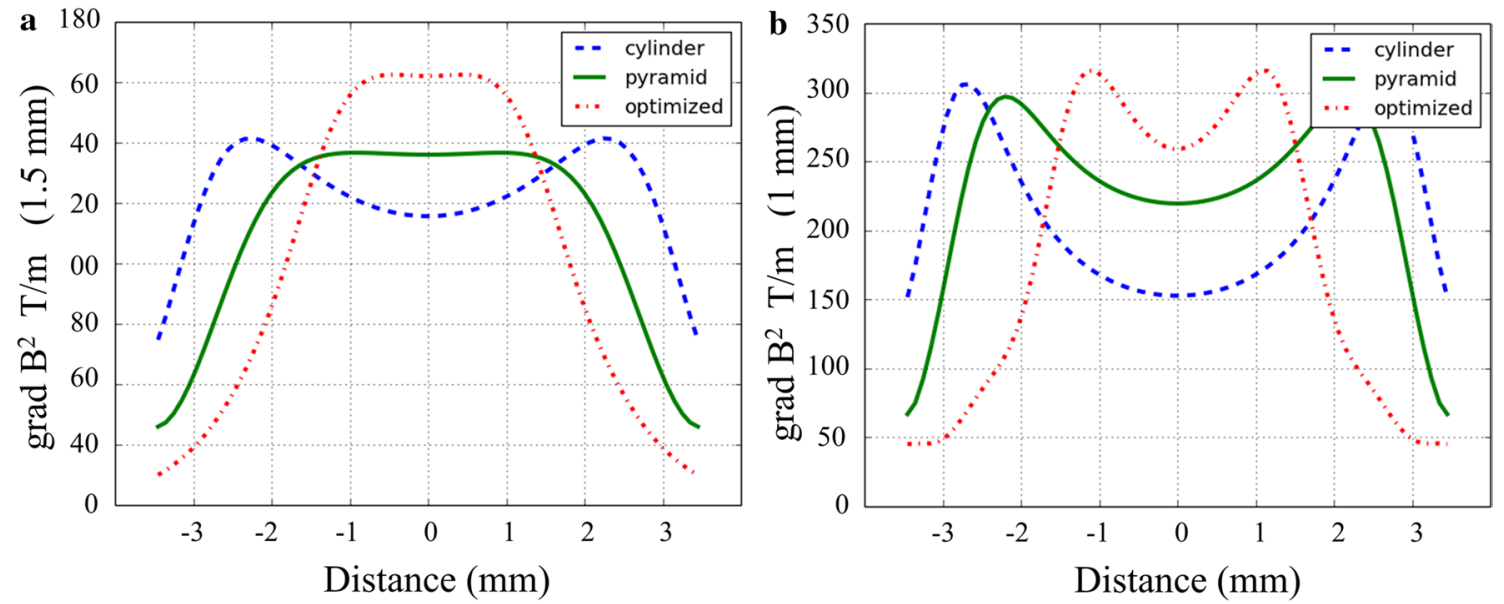

Fig. 12 Field gradients are predicted to differ between magnets of different shapes. a 2D plot of the magnetic field gradient (gradient flux density) at $1.5 \mathrm{~mm}$ along the $z$-axis. b $2 \mathrm{D}$ plot of the magnetic field gradient (gradient flux density) at $1 \mathrm{~mm}$ along the $z$-axis

in vivo, it is possible to optimize this parameter using different types of magnet arrays as demonstrated in this study and other similar studies [18].

Such numerical studies will help us to understand the principles behind time-varying magnet array-induced cytotoxicity and facilitate the optimization of this process. From a numerical perspective, the size and magnetic susceptibility of the nanoparticles used (Polymag, nTmag-100 nm), the distance between the magnet array and the cell culture plate $(1 \mathrm{~mm})$, the rapid accumulation of MNPs by the use of an alternate pole magnet array, and the variation in magnetic force mediated by a time-varying field gradient are behind the increased cytotoxicity of cancer cells.

\section{Conclusions}

Therefore, from our field measurements, numerical calculations and our cytotoxicity experiments, surface functionalized, super paramagnetic iron oxide nanoparticle at low concentrations was externally manipulated, using a timevarying field gradient technique (at very low frequencies, i.e., $1-4 \mathrm{~Hz}$ ), to induce magneto-mechanical cell death in cancer cells. This is a key to the effective inducement of minimally invasive cytotoxicity in cancer cells in physiological and clinical settings; further multidisciplinary research is required to make substantial progress in this area. 
Acknowledgements MS worked for nanoTherics limited, but currently funded by the EPSRC and the Imperial College London [EP/ N509486/1: 1979819]. JD is a consultant for nanoTherics Limited. AM, SIJ and JL are academic researchers.

Open Access This article is distributed under the terms of the Creative Commons Attribution 4.0 International License (http://creativeco mmons.org/licenses/by/4.0/), which permits unrestricted use, distribution, and reproduction in any medium, provided you give appropriate credit to the original author(s) and the source, provide a link to the Creative Commons license, and indicate if changes were made.

\section{References}

1. I.J. Bruce, T. Sen, Surface modification of MNPs with alkoxysilanes and their application in magnetic bioseparations. Langmuir 21, 7029-7035 (2005)

2. F. Scherer, M. Anton, U. Schillinger, J. Henke, C. Bergemann, A. Kruger, B. Gansbacher, C. Plank, Magnetofection: enhancing and targeting gene delivery by magnetic force in vitro and in vivo. Gen. ther. 9, 102-109 (2002)

3. M. Subramanian, J. Lim, J. Dobson, Enhanced nanomagnetic gene transfection of human prenatal cardiac progenitor cells and adult cardiomyocytes. PLoS One 8(7), .e69812 (2013)

4. S. Hapuarachchige, Y. Kato, E.J. Ngen, B. Smith, M. Delannoy, D. Artemov, Non-temperature induced effects of magnetized iron oxide nanoparticles in alternating magnetic field in cancer cells. PLoS One 11, 0156294 (2016)

5. E. Zhang, M.F. Kircher, M. Koch, L. Eliasson, S.N. Goldberg, E. Renström, Dynamic magnetic fields remote-control apoptosis via nanoparticle rotation. ACS Nano 8, 3192-3201 (2014)

6. M. Subramanian, A. Miaskowski, G. Pearce, J. Dobson, A coil system for real-time magnetic fluid hyperthermia microscopy studies. Int. J. Hyperther. 32, 112-120 (2016)

7. N.S. Satarkar, J.Z. Hilt, Magnetic hydrogel nanocomposites for remote controlled pulsatile drug release. J. Control. Release. 130, 246-251 (2008)

8. S. Dutz, Are magnetic multicore nanoparticles promising candidates for biomedical applications? IEEE Trans. Magn. 52, 1-3 (2016)

9. O. Mykhaylyk, Y.S. Antequera, D. Vlaskou, C. Plank, Generation of magnetic nonviral gene transfer agents and magnetofection in vitro. Nat. Protoc. 2, 2391-2411 (2007)
10. M. Subramanian, A.J. Tyler, E.M. Luther, E.D. Daniel, J. Lim, J. Dobson, Oscillating magnet array-based nanomagnetic gene transfection: a valuable tool for molecular neurobiology studies. Nanomaterials 7(2), 28 (2017)

11. M. Subramanian, G. Pearce, O.K. Guldu, V. Tekin, A. Miaskowski, O. Aras, P. Unak, A Pilot study into the use of FDG $\mathrm{mNP}$ as an alternative approach in neuroblastoma cell hyperthermia. IEEE Trans. Nanobiosci. 15, 517 (2016)

12. Q.A. Pankhurst, J. Connolly, S.K. Jones, J. Dobson, Applications of MNPs in biomedicine. J. Phys. D: Appl. Phys. 36, R167 (2003)

13. E.P. Furlani, K.C. Ng, Nanoscale magnetic biotransport with application to magnetofection. Phys. Rev. E 77, 061914 (2008)

14. E.P. Furlani, X. Xue, A model for predicting field-directed particle transport in the magnetofection process. Pharm. Res. 29, 1366-1379 (2012)

15. A. Garraud, C. Velez, Y. Shah, N. Garraud, B. Kozissnik, E.G. Yarmola, K.D. Allen, J. Dobson, D.P. Arnold, Investigation of the capture of magnetic particles from high-viscosity fluids using permanent magnets. IEEE Trans. Biomed. Eng. 63, 372-378 (2016)

16. L.A. Greene, A.S. Tischler, Establishment of a noradrenergic clonal line of rat adrenal pheochromocytoma cells which respond to nerve growth factor, Proc. Natl. Acad. Sci. USA 73, 2424-2428 (1976)

17. A. Fouriki, J. Dobson, Oscillating magnet array-based nanomagnetic gene transfection of human mesenchymal stem cells. Nanomedicine 9(7), 989-997 (2014)

18. L.C. Barnsley, D. Carugo, E. Stride, Optimized shapes of magnetic arrays for drug targeting applications. J. Phys. D Appl. Phys. 49(22), 225501 (2016)

19. L.C. Barnsley, D. Carugo, M. Aron, E. Stride, Understanding the dynamics of superparamagnetic particles under the influence of high field gradient arrays. Phys. Med. Biol. 62(6), 2333 (2017)

20. T. Kalwarczyk, N. Ziebacz, A. Bielejewska, E. Zaboklicka, K. Koynov, J. Szymanski, A. Wilk, A. Patkowski, J. Gapinski, H.J. Butt, R. Hołyst, Comparative analysis of viscosity of complex liquids and cytoplasm of mammalian cells at the nanoscale. Nano Lett. 11, 2157-2163 (2011)

Publisher's Note Springer Nature remains neutral with regard to jurisdictional claims in published maps and institutional affiliations. 